\title{
Summary of Embedded Systems Development
}

\author{
Xin Wang ${ }^{1, \text { a }}$, Zhong Wang ${ }^{2, \mathrm{~b}}$, Wei Huang ${ }^{3, \mathrm{c}}$, Guan Qi Wen ${ }^{4, \mathrm{~d}}$ \\ and Shao Lei Zhang ${ }^{5, e}$ \\ 1, 2, 3,4,5Xi'an Research Institute of High Technology, Xi'an, China \\ a15114988523@163.com, bdsp863wang@163.com, chuangwei_1025@163.com, \\ dkioukiou@foxmail.com, ${ }^{d}$ jianjianyuanqu11@163.com
}

Keywords: Embedded System, Embedded Processor, Embedded Operating System

\begin{abstract}
This paper discusses the concept and characteristics of embedded system, introducing the components of embedded system in detail, and introducing the processor and operating system of embedded system. This paper had a vision of future which is developing trend of embened systems through summarizing the development and the present situation for application of embedded system.
\end{abstract}

\section{Introduction}

With the rapid development of communication network technology and the large scale integrated circuit, embedded system has become a focus of IT field. At present, the product development and application degree of embedded system has become a specific standards of social informatization and intelligentize. The development of embedded system is based on the actual needs of social production and life, it combined with advanced computer technology, electronic technology and the specific application of various industries. It is a technology-intensive and innovative knowledge integration system. In this paper, reviewing of the embedded system and the basic structure of embedded systems, summarizing the current development process of embedded systems, and expecting the future development trend of embedded system.

\section{Concept and Characteristics of Embedded System}

\section{Concept of Embedded System.}

Embedded system is generally defined as a special-purpose computer, which is based on application, computer technology, software and hardware can be cut and adapted to the application system, and the functions, reliability, cost, volume and power are strictly required. ${ }^{[1]}$

Embedded system can be divided into two parts of hardware and software, hardware is mainly composed of the embedded processor and peripheral hardware, the software is composed of the embedded operating system and application software. ${ }^{[2]}$ Embedded system is a s set of hardware and software as a whole, which can independently complete a specific work of the specific computer system. Embedded systems typically perform preset tasks with specific requirements for specific devices, unlike the general-purpose computer, such as personal computers. Based on the particularity and pertinence of the embedded system, the designer can optimize it extremely, reduce the cost and improve the efficiency of the embedded system.

\section{Characteristics of Embedded System.}

The characteristics of embedded system is following:

(1) Strong specificity. Embedded system is a kind of special computer system which is oriented to a specific application and has some practical requirements. It has the characteristics of small size, low cost, low power consumption and high integration. Embedded system is user-oriented, product-oriented, application-oriented, it has a strong specificity. It need be reasonable cut according to the actual system.

(2)System simplification. Embedded systems and practical applications combined closely. 
There is no obvious difference between system software and application software, because it designed to achieve specific needs. It is not required complicated functional design and implementation, the system simplification is also conducive to control costs and improve system security.

(3)High real-time operating system. High real-time system software is the basic requirements of embedded operating system aimed at improving the speed and efficiency of the implementation of the system. Software code requires high quality and high reliability, real-time, so the software is generally solidified in the memory chip or processor internal memory.

\section{Embedded System Components}

Embedded system is divided into embedded microprocessors, peripheral hardware devices, embedded operating systems and application software. As shown in Figure 1, in the embedded system architecture, the bottom is the embedded processor core, peripheral hardware-based hardware platform. Hardware layer is above the operating system, it is responsible for the entire system hardware and software resources, scheduling, storage management, clock management and interrupt management functions. Above the operating system, it is the application software, which achieve the main function of embedded systems, and is responsible for system and user interaction. [3]

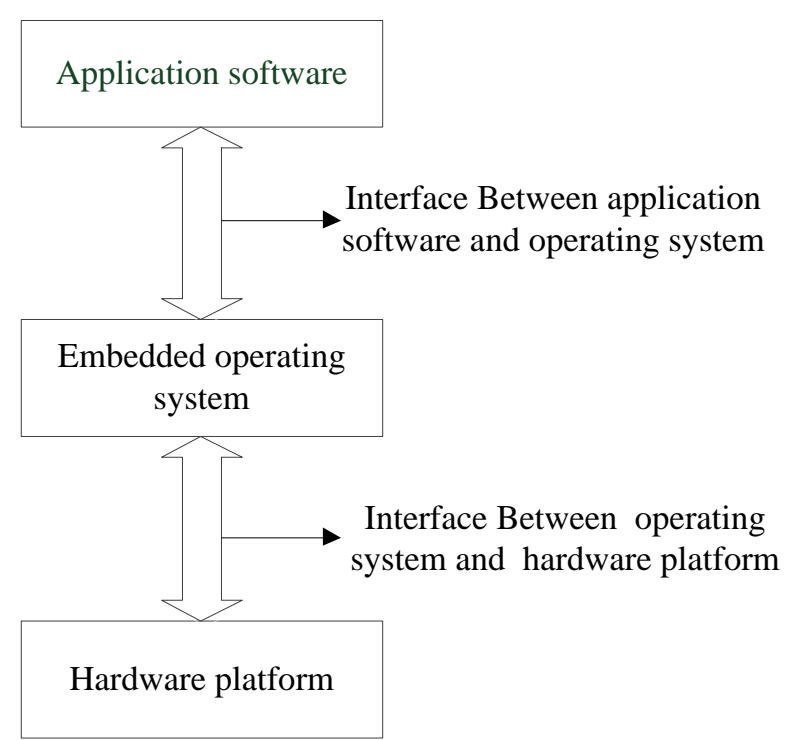

Figure 1 embedded system architecture

\section{Concept and Classification of Embedded Processor.}

An embedded controller is a control system that performs control functions independently and it has the ability to handle complex data. The embedded processor is responsible for controlling the main work of system operation and operation as the core of the embedded system. The embedded processor has the following features: real-time multitasking capability, memory protection, scalable microprocessor architecture, strong interrupt handling capability, and low power consumption for efficient and reliable system tasks. ${ }^{[4]}$ At present the common embedded processor can be divided into four kinds:

(1) Embedded MCU(MCU, Microcontroller Unit). It is the entire computer system integrated into a chip, the completion of the system's main signal control functions. Embedded MCU include ROM/ EPROM, RAM, Bus, Timer/Counter, Watchdog Timer, I/O, serial interface, A/ D, D/A and other necessary functions and peripherals.

(2) Embedded DSP(MPU , Digital Signal Processor). It is dedicated to signal processing processors, the system structure and instruction algorithm aspects of the special design. It has high efficiency and the implementation of the instruction execution speed . Embedded DSP have been widely used in digital filtering, FFT, image processing, spectrum analysis and other instruments. 
(3)Embedded MPU (MPU, MicroProcessor Unit) is a general-purpose computer from the CPU evolved, it is different from the general-purpose computer CPU. Embedded system is a specific application-oriented system, so the actual embedded Application, the embedded MPU only retained the function of hardware which is closely related to the embedded applications, to remove the other redundant parts. Compared with general-purpose computer CPU, embedded MPU has the advantages of small size, light weight, low power consumption, low cost and high reliability.

(4)Embedded SOC (SOC, System On Chip). Embedded system is the largest inclusive of integrated devices. The biggest feature of Embedded SOC system is breaking the boundaries between hardware and software. It achieves a complex system using VDHL and other hardware description language within a silicon chip. SOC has the advantage that most of the system components are in the system, so that the whole system is highly simplified, reducing the system size and power consumption, improving the efficiency of design and production.

\section{Embedded Peripheral Hardware Devices.}

Embedded peripheral hardware device is used for the completion of storage, communication, display, debugging and other auxiliary functions of other hardware devices in the embedded system hardware system, except for the central control for the embedded processor. At present, embedded peripheral hardware devices which is frequently-used can be divided into storage devices, communication devices and display devices according to their functions.

\section{Embedded Operating System.}

Embedded operating system refers to the operating system used in embedded systems. Embedded operating system is responsible for all hardware and software resources allocation, scheduling, coordination, and concurrent activities of the embedded system. ${ }^{[5]}$ Embedded operating system has become mature from simple one. The more representative operating system are VxWorks, Windows CE, QNX, Linux and so on. The main characteristics of these types of embedded operating systems are compared and analyzed as shown in Table 1.

Table 1 Embedded operating system features contrast

\begin{tabular}{|c|c|c|}
\hline $\begin{array}{c}\text { Embedded } \\
\text { Operating System }\end{array}$ & Development Agencies & Main Feature \\
\hline VxWorks & $\begin{array}{c}\text { United States } \\
\text { WindRiver company }\end{array}$ & $\begin{array}{c}\text { High real-time, High reliability, can be } \\
\text { cut }\end{array}$ \\
\hline Windows CE & Canada QNX company & $\begin{array}{c}\text { Modular, Structured, and easy to port } \\
\text { with Windows }\end{array}$ \\
\hline QNX & United States \\
Microsoft Corporation & $\begin{array}{c}\text { Operation and development is simple, } \\
\text { with good scalability }\end{array}$ \\
\hline Linux & \multicolumn{2}{|c|}{$\begin{array}{c}\text { Core small, Stable operation, High } \\
\text { Efficiency,Open source, support for } \\
\text { X86, ARM,MIPS and other structures } \\
\text { of the chip }\end{array}$} \\
\hline
\end{tabular}

\section{Embedded Application Software.}

Embedded application software is the upper layer of embedded system software, which defines the main functions of embedded devices and uses, and it is responsible for interacting with users. Embedded application software is a manifestation of embedded system functions, such as flight control software, car navigation maps, mobile software, generally for specific areas and applications. In addition to the characteristics of common general-purpose software, embedded application software has the characteristics of small size, difficult development, high real-time and reliability, 
and software-curing storage is closely related to embedded system.

\section{Development and Application of Embedded System}

In 1971, Intel Corporation issued the first single-chip 4004, after nearly four decades of developing, computer technology, electronic technology and large-scale integrated circuit had continuous progress, a wide range of embedded microprocessors, microcontrollers they all had a big Scale development and application. Embedded systems has also entered a stage of rapid development.

\section{Development Process of Embedded System.}

With the development of information, network and intelligence, embedded system has been more and more widely used. In recent decades, summary of the development process, the embedded system can be divided into the following stages of development:

(1) Early stage of embedded technology: The early embedded system is to a simple computer or microcontroller as the core, with servo, testing, equipment instructions and other functions. Most of the early embedded systems used in industrial control or aircraft and missiles and other weapons systems. This stage of the embedded system is characterized by a single system function and structure, and the processing efficiency is low, storage capacity is small, suitable for special fields.

(2) Embedded processor and embedded operating system as the symbol of embedded systems: With the development of parallel technology, pipeline, interrupt systems, operating systems and other technologies, especially in the advent of microprocessors, embedded systems has been the real developing. The main features of this stage are the emergence of embedded processors with high reliability and low power consumption, such as ARM, PowerPC and MIPS. At the same time, the embedded operating system makes the embedded system small, reliability, low power consumption and so on.

(3) Embedded system based on chip technology and Internet technology: The rapid development of large scale integrated circuit and microelectronics, SoC technology makes the embedded system become smaller, but the function is more and more powerful. The emergence of NoC (NetworkonChip), Internet and smart appliances, industrial control technology, embedded systems, network trends will make it more rapid development.

\section{Application Status of Embedded System.}

(1)Embedded system of industrial automatic control equipment has been relatively rapid development in the field of industrial control. In industrial production, the widespread application of embedded systems has become the main way to improve production efficiency and reduce human resources. such as CNC(Computerized Numerical Control) machine tools, power grid equipment monitoring, and petrochemical systems.

(2)In the field of environmental engineering and nature, such as real-time monitoring of hydrological data, real-time meteorological information collection, seismic monitoring network, flood control and soil and water monitoring, water and air pollution monitoring, because of which work involve complex natural environment and the need for long real-time information collection, embedded systems have been replaced by artificial unmanned monitoring. ${ }^{[6]}$

(3)In the field of smart household appliances, because it has broad market prospects, which will enable embedded systems to be the most widely used. At present, the household appliances became webified and intelligentize, people could control the air conditioners, refrigerators and other smart home appliances through the phone and the internet.

\section{Development Trends of Embedded System}

(1) Network become an inevitable trend. In order to meet the requirements of network development in the development of embedded devices, it is bound to provide hardware on the network communication interface support. Embedded development is very rapidly, not only the requirements of embedded devices can connect to the Internet, but also interconnect needs of embedded devices to achieved internet of things by embedded devices, so that the human society 
and the information network will enter into a new stage.

(2) Data sharing. With the popularity of embedded devices, a variety of embedded devices will inevitably produce a lot of data, so how to integrate these data, sorting, co-ordination analysis, embedded devices have become the development process of new requirement. With the result of a number of new embedded system application requirements,such as smart management system for home appliances which can intelligently control all household appliances, traffic data management system which can comprehensively analyze the data detected by all the urban road monitoring equipment in one city and so on. How to realize data sharing among all embedded devices, and analyze and manage these data will be a new research topic in the future development of embedded systems. ${ }^{[7]}$

(3) Human-computer interaction is more intelligent. With the development and application of various new technologies, such as fingerprint recognition, iris recognition, heart rate detection, speech recognition, image recognition. The application of these technologies make smart phones, smart wear equipment and other embedded devices had a new increase in the aspects of unlock the security technology and human-computer interaction experience. And with the continuous development of science and technology, the human-computer interaction interface of embedded device will become more and more intelligent in the future, the good experience for users and application requirements will be the key in the embedded devices market competition.

\section{Conclusion}

The characteristics of special nature, encapsulation, real-time and reliability make embedded device has been widely used. embedded systems will eventually become small, special, intelligent because of the continuous product for new technologies and huge demanding of market made embedded system developed rapidly. How to meet new needs produced in life, and improve work efficiency and quality of life, will be the development of embedded systems direction in the future.

\section{References}

[1] Dutt N, Jantsch A, Sarma S. Toward Smart Embedded Systems: A Self-aware System-on-Chip (SoC) Perspective[J]. Acm Transactions on Embedded Computing Systems, 2016, 15(2):1-27.

[2] Hsiung $\mathrm{P}$ A, Kuo $\mathrm{T} \mathrm{W}$, Chang $\mathrm{Y} \mathrm{H}$, et al. Introduction to the special issue on reconfigurable cyber-physical and embedded system design[J]. Journal of Systems Architecture, 2016, 62:39-39.

[3] Osamnia M, Okada H, Berena A J, et al. A novel automated course generation system embedded in the online lecturing platform for higher education[J]. Computer Applications in Engineering Education, 2016, 24(4):586-598.

[4] Cimatti A, Tonetta S. Contracts-refinement proof system for component-based embedded systems[J]. Science of Computer Programming, 2015, 97:333-348.

[5] Schmidt J S, Jenkinson M E. Operating system and architecture for embedded system:, US9075671[P]. 2015.

[6] Samantaray A K. Development of a Real-time Embedded System for Speech Emotion Recognition[J]. 2014.

[7] Sivasankaran V, Muruganand S, Periasamy A. Advanced Embedded System Assisted Gsm And Rfid Based Smart School Management System[J]. International Journal of Advanced Research in Electrical Electronics \& Instrumentation Engineering, 2013, 2(7).

[8] Paul, Soulier, Depeng, et al. A Survey of Language-Based Approaches to Cyber-Physical and Embedded System Development[J]. Journal of Tsinghua University (Science and Technology), 2015, 20(2):130-141. 